\title{
PEDIATRIC DENTIST ACCESSIBILITY AND POST-OPERATIVE COMPLICATIONS OF LARYNGEAL MASK AIRWAY VERSUS NASOTRACHEAL INTUBATION IN FULL MOUTH REHABILITATION UNDER GENERAL ANAESTHESIA: A RANDOMISED CONTROLLED TRIAL
}

\author{
Ahmed Elkhadem*, Mohamed Abdel-Ghany ${ }^{* *}$ and Passant Nagi*
}

\begin{abstract}
Background: Nasotracheal intubation (NTI) is the standard used technique for treating children's teeth under general anaesthesia (GA). Nevertheless, it may cause adverse effects like laryngeal pain, post-operative nausea and vomiting (PONV), and dysphonia. Hence, this study aimed to compare the pediatric dentist's access to the mouth, PONV, laryngeal pain, dysphonia, patients' recovery time, parents satisfaction and operators satisfaction for children received NTI or an LMA.
\end{abstract}

Materials and methods: Fifty children were randomised to either NTI or LMA groups. One expert pediatric dentist performed full mouth rehabilitation for all children. An expert anaesthetist used $8 \%$ sevoflurane mask for induction, followed by airway management either with LMA or NTI. Muscle relaxant was not administered for both groups to permit lung muscles to work normally during operation.

Results: The baseline characteristics were similar between NTI and LMA with no statistically significant difference. Regarding the postoperative laryngeal pain and dysphonia, LMA showed less risk of occurrence with a relative risk of 0.27 (95\% CI: $0.1,0.69)$ and 0.22 (95\% CI: $0.1,0.5$ ) respectively, and these results were statistically significant $(\mathrm{P}<0.01)$. On the other hand, the use of NTI decreased the total operation time by 23 minutes (95\% CI:14.8, 31.2). Besides, NTI resulted in better intraoral accessibility for the pediatric dentist with $92 \%$ rating as excellent, while LMA was obstructing the view in $40 \%$ of cases and prevented working efficiently in $32 \%$ of cases. There were no statistically significant differences between the two groups in the incidence of PONV, dental pain scores, recovery time, or parents' satisfaction level ( $\mathrm{P}>0.05)$.

Conclusion: For this group of Egyptian children who underwent full-mouth dental rehabilitation under general anaesthesia, the LMA provided less risk for postoperative laryngeal pain and dysphonia. However, the pediatric dentist had better accessibility and workability with NTI, which subsequently decreased the operation time significantly.

KEYWORDS: Laryngeal mask airway; Nasotracheal intubation; Full mouth rehabilitation; General anaesthesia; Pediatric dentist accessibility

* Lecturer in the Pediatric Dentistry Department, Faculty of Dentistry, Cairo University, Egypt.

** Lecturer in Anesthesiology Department, Faculty of Medicine, Cairo University, Egypt. 


\section{INTRODUCTION}

The combination of general anaesthesia (GA) and dental treatment procedures are required with uncooperative children or with the need for surgical procedure as in cases of trauma to the face or jaws. General anaesthesia may also be indicated if the patient has a physical or mental disability or possess grave fear and anxiety for dental procedures ${ }^{[1-8]}$. In full mouth rehabilitation, general anaesthesia decreases the psychological and physical stress for all parties included namely the child, the parents and the pediatric dentist. However, there are some undesirable effects of GA, including transient incidents of postoperative nausea and vomiting (PONV), dizziness, headache, and agitation; or more severe complications as respiratory depression, respiratory arrest, laryngospasm, and anaphylaxis ${ }^{[9,10]}$.

Nasotracheal intubation (NTI) is considered the most commonly used airway management technique in general anaesthesia for pediatric dental procedures. For dental practitioners, NTI provides an unobstructed view and enlarges work field in the mouth, which consequently facilitates instrument insertion and handling ${ }^{[1]}$. However, NTI has some drawbacks which include slow recovery period and postoperative coughing, dysphonia and laryngeal pain ${ }^{[1,2]}$.

The laryngeal mask airway (LMA) is easily inserted and removed, though it may be a useful alternative to NTI in children. However, for the experienced anaesthetist, there would be no difficulties in the insertion of both NTI or LMA. The LMA has better patient tolerance, and a lower risk of upper respiratory infection ${ }^{3}$ which is attributed to the ease of LMA insertion without using a muscle relaxant, especially in conditions where an adequate depth of anaesthesia suppresses airway reflexes. Postoperative complications like nausea, vomiting and sore throat are less frequent with the LMA than with NTI. Further, it was found that laryngeal pain during the first postoperative hours in adults accompanied the use of an endotracheal tube ${ }^{[11,12]}$.

This trial aimed to compare the LMA and NTI use in full-mouth dental treatment under general anaesthesia in terms of postoperative complications for the patient, and accessibility of the dental field for the pediatric dentist.

\section{MATERIALS AND METHODS}

The current study was a prospective, doubleblinded, randomised controlled trial, with parallel arms. Informed written consent was derived from the legal guardians of all children who participated in this study.

The sample size in the current study was determined by a computer software PSS (the power and sample size calculation) to be 25 children per group. The variable used for sample size calculation were derived from a previous study ${ }^{[1]}$ with a minimal clinical difference of 1.5 , an alpha level 0.05 with a power of 95\%. Furthermore, using (SPSS 20.0; IBM Corporation, USA)

Fifty children who attended Mira Dental pediatric unit (Cairo, Egypt) participated in the current study aged 3-7 years. They were recruited between October 2018 and January 2019 for dental caries treatment under GA. Exclusion criteria included mental retardation, pulmonary, cardiac or vascular disease, enlarged tonsils, and legal guardian who refused the participation of their children in the study. Participants' parents were permitted to attend the beginning of the induction through a gas mask before the start of intubation.

Random sequence generation was performed by a statistician (ED). Each group comprised 25 patients who were randomly assigned to one of the two groups (either NTI or LMA). Parent/guardian selected a dark sealed opaque envelope immediately before the operation to assign the participant to the designed group. A secretary (EM) in the dental centre implemented the randomisation process. 


\section{Anaesthesia protocol for NTI group:}

The same anesthesiologist (MA) intubated all patients in the NTI or LMA groups. For all children undergoing treatment, mask induction was carried out for one minute using $8 \%$ sevoflurane and $100 \%$ oxygen. After the loss of patient consciousness, an intravenous line was established by the anesthesiologist to administer $2 \mathrm{mg} / \mathrm{kg}$ of $1 \%$ propofol (Propofol-Lipuro ${ }^{\circledR}$ Germany). After selecting the nasotracheal tube size using $([$ age $/ 4]+3.5)$ formula, NTI was done with a cuffed tube into the airway after lubrication with water-soluble jelly. Following intubation, a medical tape was used to secure the nasotracheal tube. After that, a pharyngeal pack $30 \mathrm{~cm}$ in length was moistened and inserted to avoid inhalation of blood or solid object during the operation.

\section{Anaesthesia protocol for LMA group}

The same mask induction procedures in NTI were performed by the same anaesthesiologist (MA). The laryngeal cuff was fully deflated before insertion. Once deflated, a water-based soluble jelly was used to lubricate the backside of the cuff. The LMA is pushed inside the child's mouth until it is felt seated in the pharynx over the supraglottic area. Child's weight was the determining factor in selecting the LMA [13]. We used sizes 1.5, 2 and 2.5 for children weighing 5-10 kg, 10-20 kg and 20-30 $\mathrm{kg}$ respectively. The LMA was inflated according to the manufacturer's instructions after insertion.

For all children, no muscle relaxant was used in either of the two groups. Also, for all children, anaesthesia was maintained with $10 \%$ isoflurane in $100 \%$ of oxygen. Fifteen minutes before the end of the surgery, both groups received Tenoxicam (Eipico, Egypt) $0.4 \mathrm{mg} / \mathrm{kg}$ intravenously for analgesia and Metoclopramide (Primeperan, Sanofi, Egypt) $0.4 \mathrm{mg} / \mathrm{kg}$ intravenously to minimise PONV ${ }^{[14]}$. Patient monitoring was performed using electrocardiography plus noninvasive arterial blood pressure.

\section{Dental treatment procedures}

A single experienced pediatric dentist (AE performed all the dental procedures. The LMA was secured to the corner of the opposite side of the mouth using adhesive tape, thus making the planned operative side of the oral cavity accessible. After the pediatric dentist finished restorative treatment of upper and lower right quadrant, the anaesthetist relocated LMA to the left quadrant of the mouth. Meanwhile, there was no stoppage time in NTI group since both sides of the oral cavity are accessible to the dentist at all times.

Dental procedures performed included composite restoration for occlusal caries, stainless steel crowns for proximal decay with or without pulp involvement, endodontic procedures (pulpotomy, pulpectomy) and finally extraction for unrestorable teeth.

The deft and DMFT caries indices and the number of teeth treated were recorded. Further, we calculated the duration of both dental operation and anaesthesia in minutes using a stopwatch. Once all dental treatment was completed, and protective airway reflexes were regained,LMA or endotracheal tube was removed, and patients were transferred to the recovery room. Post-discharge instructions included rest throughout the day and an NSAID was prescribed for 48 hours.

\section{Outcomes}

At the recovery room, a pedodontist (P.N) started to evaluate patients every five minutes. The physical status of children recovering from general anaesthesia was assessed using the Aldrete system. Aldrete system has five categories with a score of 0-2 each (consciousness, activity, respiration, circulation, $\mathrm{O}_{2}$ saturation) thus, the maximum score expected is $10^{[15]}$. Recovery time was started once the child arrived at the recovery room till the child scored $\geq$ nine at Aldrete system ${ }^{[16]}$ and could answer the pedodontist. 
An expert pedodontist (P.N) who was blinded to the airway management technique, recorded postoperative discomfort. Furthermore, the pedodontist (P.N) recorded the following outcomes 1-hour postoperative: dental and laryngeal pain, dysphonia, and PONV as binary outcomes with either the absence or presence of the event. Further, P.N telephoned the parents after 24 hours to record the incidence of PONV throughout.

The satisfaction level of parents or guardians was self-graded on scales from (0 to 10), where zero is very dissatisfied, and ten is very satisfied. Also, the access to the oral cavity working area was graded by the operating pedodontist (A.E) as; excellent, obstructed the view but able to operate, or poor access with an obstructed view. The pediatric dentist was not blinded to the anaesthesia technique being used.

\section{Statistical analysis}

Statistical analysis was performed. KolmogorovSmirnov test for data normality and to test differences in baseline characteristic, the statistician used the two-sample t-tests. An 0.05 cut off level was used for statistical significance. Relative risk and $95 \%$ confidence intervals were used for binary outcomes.

\section{RESULTS}

At the end of the study, 50 children participated with male to female ratio $1: 1$ and a mean age of $4.6 \pm 1.2$ years (Table 1). A flow chart represents the recruitment of the patients and their followup (Figure 1). Baseline characteristics of the two groups were nearly equal, with no significant differences ( $\mathrm{P}>0.05$; Table 1). Those characters included; age, gender, weight, deft values, and the number of dental procedures.

The outcomes were divided into patient-oriented endpoints and dentist-oriented ones (Table 2). The patient-oriented parameters assessed four postoperative complications, namely laryngeal pain, dental pain, dysphonia and PONV. Besides, parental satisfaction following the GA was recorded. Meanwhile, dentist endpoints included intraoral accessibility for the pediatric dentist, total dental operating time and recovery time.

In terms of dental pain and PONV, there was no statistically significant difference between LMA and NTI groups with a relative risk of $0.75(95 \% \mathrm{CI}$ $0.3,1.85)(\mathrm{p}=0.54)$ and $0.71(95 \% \mathrm{CI} 0.26,1.95)$ $(\mathrm{p}=0.69)$. On the other hand, the risk of laryngeal pain was less in LMA compared to NTI with a relative risk reduction of 0.73 (95\% CI $0.31,0.89)$ $(\mathrm{p}=0.03)$. Further, the risk of dysphonia was less in LMA compared to NTI with a relative risk reduction of $0.77(95 \% \mathrm{CI} 0.49,0.89)(\mathrm{p}=0.01)$. As for parental satisfaction, there was no statistically significant difference between LMA and NTI groups.

Regarding dentist-oriented endpoints, NTI was superior compared to LMA with an excellent rating of $92 \%$ and $28 \%$ respectively. Moreover, the use of NTI reduced the total dental operation time by 23 minutes $(95 \%$ CI $14.8,31.2)(\mathrm{p}<0.01)$, and this result was statistically significant. Meanwhile, the child's recovery time was nearly similar in both groups ranging between 10 and 11 minutes for LMA and NTI groups, respectively, and this result was statistically insignificant. 
TABLE (1) Demographics, operation and anaesthesia times, and deft values of the patients

\begin{tabular}{|l|c|c|c|}
\hline \multicolumn{1}{|c|}{ Demographic and clinical characteristics } & NTI (n=25) & LMA (n=25) & P-value \\
\hline Age (years) & $4.5 \pm 1.2$ & $4.7 \pm 1.3$ & 0.11 \\
\hline Gender: male/female & $12 / 13$ & $13 / 12$ & 0.81 \\
\hline Weight (kg) & $18.7 \pm 3.5$ & $19.8 \pm 4.6$ & 0.13 \\
\hline Deft (decayed/indicated for extraction/ filled teeth) & $10.2 \pm 3.7$ & & \\
\hline Number of dental procedures & & 3.4 & $0.1 \pm 2.5$ \\
\hline Pulpotomy + stainless steel crown crown & $6.7 \pm 1.9$ & $1.4 \pm 0.4$ & 0.36 \\
\hline Composite restoration & $0.71 \pm 0.2$ & $1.1 \pm 0.2$ & 0.14 \\
\hline Extraction & $1.7 \pm 0.3$ & $3.2 \pm 0.7$ & 0.34 \\
\hline Pulpectomy + esthetic crown & $3.1 \pm 0.8$ & & 0.4 \\
\hline
\end{tabular}

TABLE (2) Comparison of LMA versus NTI in terms of patient-oriented and dentist-oriented endpoints.

\begin{tabular}{|c|c|c|c|c|}
\hline & LMA, n (\%) & NTI, n (\%) & RR $(95 \% \mathrm{CI})$ & P-value \\
\hline Laryngeal pain & $4(16)$ & $15(60)$ & $0.27(0.1,0.69)$ & $0.00^{*}$ \\
\hline Dental pain & $6(24)$ & $8(32)$ & $0.75(0.3,1.85)$ & 0.36 \\
\hline Dysphonia & $5(20)$ & $22(88)$ & $0.22(0.1,0.5)$ & $0.00 *$ \\
\hline PONV & $5(20)$ & $7(28)$ & $0.71(0.26,1.95)$ & 0.2 \\
\hline Parent satisfaction (0-10) & $8.1 \pm 2.5$ & $6.7 \pm 2.9$ & $-1.4(-2.94,0.14)$ & 0.36 \\
\hline \multicolumn{5}{|c|}{ Intraoral accessibility for pediatric dentist } \\
\hline Excellent & $7(28)$ & $23(92)$ & $0.3(0.16,0.57)$ & $0.00 *$ \\
\hline Obstructing view but able to work & $10(40)$ & $2(8)$ & $5(1.22,20.54)$ & 0.24 \\
\hline $\begin{array}{l}\text { Poor (Obstructing view and not able } \\
\text { to work efficiently) }\end{array}$ & $8(32)$ & $0(0)$ & & \\
\hline Total dental operation time (min) & $85.7 \pm 15.9$ & $62.7 \pm 12.8$ & $-23(-14.8,-31.2)$ & $0.00 *$ \\
\hline Recovery time (min) & $10.1 \pm 3.7$ & $11 \pm 2.7$ & $0.9(-0.94,2.74)$ & 0.2 \\
\hline
\end{tabular}

Abbreviations: LMA: laryngeal mask airway; NTI: nasotracheal intubation; PONV: postoperative nausea and vomiting. 


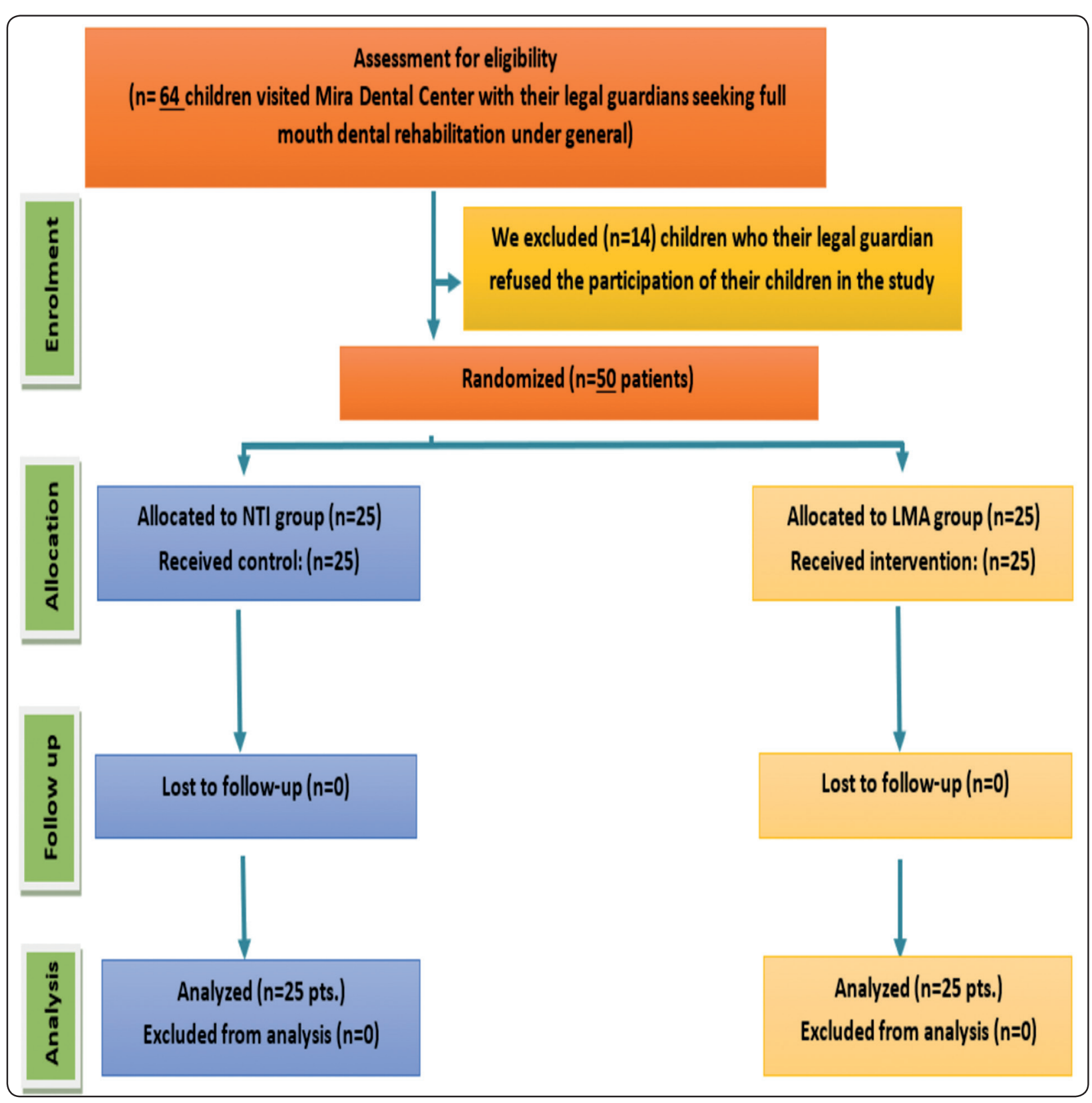

Fig. (1) Participant flow diagram

\section{DISCUSSION}

General anaesthesia is considered a magic tool for the pediatric dentist in treating children with multiple decayed teeth. When the child's fear, anxiety and involuntary movements are reduced, the pediatric dentist can provide better and less stressful dental care in a shorter time ${ }^{[17]}$. Further, GA separates the parents from the child. Thus, This study compared these two techniques during eliminating the parental anxiety during treatment which requires effort from the pediatric dentist to address during the dental treatment and in many instances, requires separating the parent from the child.

Two commonly used airway management during GA are nasal intubation or laryngeal mask airway. 
full mouth rehabilitation in children to discover their advantages and adverse effects. The outcomes measured were five patient-oriented endpoints and three dentist-oriented ones.

Patient-oriented endpoints included four post-operative complications as laryngeal pain, dysphonia (difficulty in speaking), dental pain, and PONV ${ }^{[18]}$. The fifth outcome was parental satisfaction. Laryngeal pain and dysphonia were significantly decreased in children receiving LMA as it reduces the incidence of intubation induced soft tissue trauma $[19,20]$. It should be noted that LMA doesn't require passage of a tube through the nasotracheal airway as NTI does. Instead, an inflated cuff in the LMA provides anaesthetic solution and protects the airway at the same time ${ }^{[19]}$. In previously published studies, it was found that the postoperative sore throat was more frequent in NTI when compared to LMA ${ }^{[21-23]}$. Moreover, LMA has previously shown to have reduced the incidence of postoperative voice problems ${ }^{[24]}$. Nevertheless, Lalwani et al., ${ }^{[25]}$ found that there was no dysphonia after removal of either LMA or NTI in patients who underwent elective ophthalmological or lower abdominal surgery.

Meanwhile, there was no difference in postoperative dental pain between the two groups. Thus, the type of airway management during dental treatment doesn't affect the postoperative dental pain, which would be affected by the dental procedure rather than airway management persee. Further, the incidence of PONV was similar in NTI and LMA groups. Postoperative nausea and vomiting might be more affected by the anaesthetic drug used ${ }^{[26-28]}$, or the type of surgery performed rather than by the airway management technique. It should be noted that all children in both groups received an antiemetic intravenously during the operation, which might explain the decreased incidence of nausea and vomiting in both groups. Furthermore, parental satisfaction was nearly similar in both groups.
In dental treatment, the tube for airway management in GA can be placed either through the nose or the oral cavity. Nasal intubation allows the pediatric dentist to check the occlusion following placement of preformed crowns ${ }^{[18]}$. Moreover, since the anaesthetic tube is passing through the nose, the oral cavity is kept clear during airway management, providing better visibility and accessibility for the pediatric dentist. This hypothesis has been proven in our study. The intraoral accessibility for the pediatric dentist was rated as excellent in $92 \%$ of NTI cases and $28 \%$ in LMA cases. In $40 \%$ of LMA cases, the pediatric dentist suffered an obstructed view and in $32 \%$ of the cases suffered obstructed view that prevented him from doing the dental treatment properly. In 15 cases of LMA group, the pediatric dentist found difficulty in placing a bite block to open the mouth because the tube is positioned in the same non-working side. Thus, the dentist worked without full mouth opening, which added extra stress due to limited space for intraoperative work and limited visibility.

Regarding the length of dental operation, NTI decreased the time needed for full mouth rehabilitation in children by 23 minutes. Taking into consideration that no significant difference was observed between the number of restorative treatments between both trial groups, we can assume that the LMA makes it more challenging to work within the mouth. The dental operation time increased in the LMA group because of the obstructed view and decreased intraoral accessibility. However, our results are different than those reported by Zhao et al. ${ }^{[12]}$ where they found no difference in total operation time between NTI and LMA. It should be noted that they didn't record the type of dental treatment received nor their number, unlike our research which we recorded them.

A previous study linked the prolonged recovery time of children to the use of muscle relaxant during the induction to facilitate tracheal intubation ${ }^{[29]}$. 
In this study, muscle relaxants were not used, and the child was breathing autonomously under general anaesthesia which might explain the shortened recovery time ${ }^{[30]}$.

\section{Study limitations:}

The first limitation might be the short follow-up time frame, but that could be explained by the short stay of the child post-operatively in the recovery room. The second limitation is the wide range of selfreported scores of pain because of the broad range of 3 to 7 years in children included in the study.

\section{CONCLUSION}

In children who participated in the current study, the use of an LMA resulted in less postoperative laryngeal pain and dysphonia, while NTI resulted in better intraoral accessibility and decreased total treatment time significantly. Since laryngeal pain and dysphonia are transient postoperative complications, the choice for NTI in full mouth rehabilitation would be preferred because of better visibility and workability, resulting in quality dental care.

\section{DISCLOSURE}

The authors disclose that there is no financial interest with any of the material used in the study

\section{REFERENCES}

1. Knapp, R., et al., Change in children's oral health-related quality of life following dental treatment under general anaesthesia for the management of dental caries: a systematic review. Int J Paediatr Dent, 2017. 27(4): p. 302-312.

2. Dziedzic, A., The Role of General Anaesthesia in Special Care \& Paediatric Dentistry; Inclusion Criteria and Clinical Indications. SAAD Dig, 2017. 33: p. 48-54.

3. Tsukamoto, M., T. Hitosugi, and T. Yokoyama, Flexible laryngeal mask airway management for dental treatment cases associated with difficult intubation. J Dent Anesth Pain Med, 2017. 17(1): p. 61-64.

4. Chang, J. and H.Y. Kim, Prognostic factors of single-visit endodontic and restorative treatment under general anaesthesia for special needs patients. J Oral Rehabil, 2017. 44(2): p. 96-104.
5. Mallineni, S.K. and C.K. Yiu, A retrospective review of outcomes of dental treatment performed for special needs patients under general anaesthesia: 2-year follow-up. ScientificWorldJournal, 2014. 2014: p. 748353.

6. de Nova-Garcia, M.J., et al., Program for coordinated dental care under general anaesthesia for children with special needs. Med Oral Patol Oral Cir Bucal, 2007. 12(8): p. E569-75.

7. de Nova Garcia, M.J., et al., Criteria for selecting children with special needs for dental treatment under general anaesthesia. Med Oral Patol Oral Cir Bucal, 2007. 12(7): p. E496-503.

8. Prabhu, N.T., J.H. Nunn, and D.J. Evans, A comparison of costs in providing dental care for special needs patients under sedation or general anaesthesia in the North East of England. Prim Dent Care, 2006. 13(4): p. 125-8.

9. Prasanna, D. and S. Bhat, Nasotracheal Intubation: An Overview. J Maxillofac Oral Surg, 2014. 13(4): p. 366-72.

10. Li, L.Q., et al., Effects of dexamethasone on post-operative cognitive dysfunction and delirium in adults following general anaesthesia: a meta-analysis of randomised controlled trials. BMC Anesthesiol, 2019. 19(1): p. 113.

11. Radu, A.D., et al., Pharyngo-laryngeal discomfort after breast surgery: comparison between orotracheal intubation and laryngeal mask. Breast, 2008. 17(4): p. 407-11.

12. Zhao, N., F. Deng, and C. Yu, Anesthesia for pediatric daycase dental surgery: a study comparing the classic laryngeal mask airway with nasal trachea intubation. J Craniofac Surg, 2014. 25(3): p. e245-8.

13. Tian, Y., et al., A clinical trial evaluating the laryngeal mask airway-Supreme in obese children during general anesthesia. Arch Med Sci, 2017. 13(1): p. 183-190.

14. Tjia, I., M. Dalton, and M. Watcha, Management of nausea and vomiting postoperatively pediatric patients. Postoperative Nausea and Vomiting: a practical guide. Cambridge University Press, 2016: p. 119-130.

15. Aldrete, J.A. and D. Kroulik, A postanesthetic recovery score. Anesth Analg, 1970. 49(6): p. 924-34.

16. Chung, F., Discharge criteria--a new trend. Can J Anaesth, 1995. 42(11): p. 1056-8.

17. Chen, Y.P., et al., A 10-year trend of dental treatments under general anesthesia of children in Taipei Veterans General Hospital. J Chin Med Assoc, 2017. 80(4): p. 262-268. 
18. Wong, A., et al., Reducing nasopharyngeal trauma: the urethral catheter-assisted nasotracheal intubation technique. Anesth Prog, 2011. 58(1): p. 26-30.

19. Jamil, S.N., et al., A Study of the Use of Laryngeal Mask Airway (LMA) in Children and its Comparison with Endotracheal Intubation. Indian J Anaesth, 2009. 53(2): p. 174-8.

20. Ashkenazi, M., S. Blumer, and I. Eli, Post-operative pain and use of analgesic agents in children following intrasulcular anaesthesia and various operative procedures. $\mathrm{Br}$ Dent J, 2007. 202(5): p. E13; discussion 276-7.

21. Needleman, H.L., et al., Postoperative pain and other sequelae of dental rehabilitations performed on children under general anesthesia. Pediatr Dent, 2008. 30(2):p. 111-21.

22. Yu, S.H. and O.R. Beirne, Laryngeal mask airways have a lower risk of airway complications compared with endotracheal intubation: a systematic review. J Oral Maxillofac Surg, 2010. 68(10): p. 2359-76.

23. Venugopal, A., R.M. Jacob, and R.C. Koshy, A randomized control study comparing the pharyngolaryngeal morbidity of laryngeal mask airway versus endotracheal tube. Anesth Essays Res, 2016. 10(2): p. 189-94.

24. Abdi, W., et al., Sparing the larynx during gynecological laparoscopy: a randomized trial comparing the LMA Supreme and the ETT. Acta Anaesthesiol Scand, 2010.54(2): p. 141-6.
25. Lalwani, J., et al., ProSeal laryngeal mask airway: An alternative to endotracheal intubation in paediatric patients for short duration surgical procedures. Indian J Anaesth, 2010. 54(6): p. 541-5.

26. Bourdaud, N., et al., Development and validation of a risk score to predict the probability of postoperative vomiting in pediatric patients: the VPOP score. Paediatr Anaesth, 2014. 24(9): p. 945-52.

27. Eberhart, L.H., et al., The development and validation of a risk score to predict the probability of postoperative vomiting in pediatric patients. Anesth Analg, 2004. 99(6): p. 1630-7, table of contents.

28. Apipan, B., D. Rummasak, and N. Wongsirichat, Postoperative nausea and vomiting after general anesthesia for oral and maxillofacial surgery. J Dent Anesth Pain Med, 2016. 16(4): p. 273-281.

29. Julien-Marsollier, F., et al., Muscle relaxation for tracheal intubation during paediatric anaesthesia: A meta-analysis and trial sequential analysis. Eur J Anaesthesiol, 2017. 34(8): p. 550-561.

30. Ledowski, T., Muscle Relaxation in Laparoscopic Surgery: What is the Evidence for Improved Operating Conditions and Patient Outcome? A Brief Review of the Literature. Surg Laparosc Endosc Percutan Tech, 2015. 25(4): p. 281-5. 